This item was submitted to Loughborough's Research Repository by the author.

Items in Figshare are protected by copyright, with all rights reserved, unless otherwise indicated.

\title{
Effect of dilute $\mathrm{CHO}$ beverages on performance in cool and warm environments
}

PLEASE CITE THE PUBLISHED VERSION

http://dx.doi.org/10.1249/MSS.0b013e31822dc5ed

PUBLISHER

Lippincott, Williams \& Wilkins (@ American College of Sports Medicine)

VERSION

AM (Accepted Manuscript)

LICENCE

CC BY-NC-ND 4.0

REPOSITORY RECORD

Watson, Phillip, Susan M. Shirreffs, and Ronald J. Maughan. 2019. "Effect of Dilute CHO Beverages on Performance in Cool and Warm Environments". figshare. https://hdl.handle.net/2134/11377. 
This item was submitted to Loughborough's Institutional Repository (https://dspace.lboro.ac.uk/) by the author and is made available under the following Creative Commons Licence conditions.

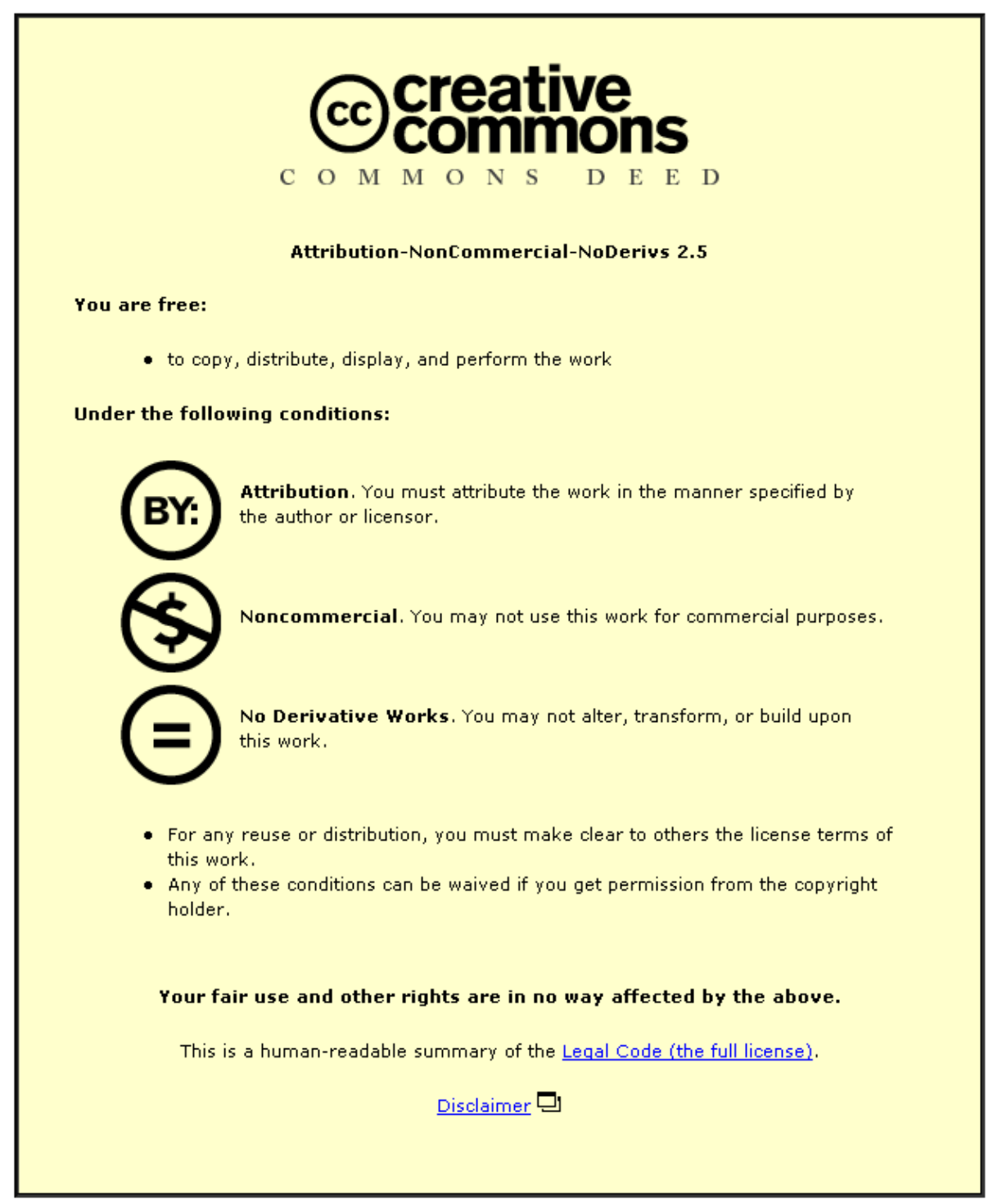

For the full text of this licence, please go to: http://creativecommons.org/licenses/by-nc-nd/2.5/ 
Effect of dilute carbohydrate beverages on performance in cool and warm environments

Watson P, Shirreffs SM, Maughan RJ.

School of Sport, Exercise and Health Sciences, Loughborough University, Leicestershire, LE11 3TU, UK.

Correspondence: Professor Ronald J Maughan,

School of Sport and Exercise Sciences, Loughborough University, Leicestershire, LE11 3TU, United Kingdom.

Telephone: $\quad$ +44 (0)1509 226329

Fax: $\quad$ +44 (0)1509226301

Email: $\quad$ r.j.maughan@lboro.ac.uk

Running title: Carbohydrate and exercise performance

External funding: This study was funded by The Coca-Cola Company. 


\section{Abstract}

Purpose: This evaluated the efficacy of drinks containing low concentrations of carbohydrate (CHO; 2-6\%) on physical performance in cool and warm environments. Methods: In two separate, but related studies, 24 healthy males completed a familiarisation trial and four trials to volitional exhaustion (TTE) at 70\% VO2max in cool conditions $\left(10^{\circ} \mathrm{C}, \mathrm{n}=12\right)$ or $60 \%$ VO2max in a warm environment $\left(30^{\circ} \mathrm{C}, \mathrm{n}=\right.$ 12). Subjects ingested $0 \%, 2 \%, 4 \%$ or $6 \%$ CHO solutions (sucrose, glucose and fructose in a ratio of 50:25:25) immediately before exercise and every 10 minutes during exercise. Results: TTE in $10^{\circ} \mathrm{C}$ was $102.6 \pm 33.9$ minutes, $109.2 \pm 33.9$ minutes, $121.0 \pm 25.7$ minutes and $122.4 \pm 29.9$ minutes in the $0 \%, 2 \%, 4 \%$ and $6 \%$ trials respectively $(P=0.012)$. Compared to the $0 \%$ trial, TTE was longer on the $4 \%$ $(\mathrm{P}=0.032 ; \mathrm{ES} 0.72)$ and $6 \%(\mathrm{P}=0.044 ; \mathrm{ES} 0.66)$ trials. In addition, TTE was longer on the $6 \%$ trial than the $2 \%$ trial $(\mathrm{P}=0.025)$. TTE was also significantly influenced by drink $\mathrm{CHO}$ content at $30^{\circ} \mathrm{C}(0 \% 94.5 \pm 24.5$ minutes, $2 \% 104.1 \pm 20.1$ minutes, $4 \%$ $105.5 \pm 26.7$ minutes, $6 \% 112.0 \pm 28.7$ minutes; $\mathrm{P}=0.046)$. No differences in TTE were apparent between $0 \%$ and the $2 \%$ or $4 \%$ trials, but TTE was longer on the $6 \%$ compared to the placebo ( $\mathrm{P}=0.045$; ES 0.62). Heart rate, core temperature or rates of substrate oxidation were not affected by drink CHO content. Conclusions: These results demonstrate significant improvements in exercise capacity over the placebo trial when $4 \%$ and $6 \% \mathrm{CHO}$ solutions were ingested at $10^{\circ} \mathrm{C}$ and a $6 \% \mathrm{CHO}$ drink at $30^{\circ} \mathrm{C}$.

Key words: prolonged exercise, time to exhaustion, heat stress, sports drinks 


\section{Introduction}

Paragraph 1 The ingestion of exogenous carbohydrate (CHO) before and during exercise undertaken in temperate environments has been demonstrated to enhance performance when the exercise duration is greater than 60 minutes $(3,8,18,19,31,39)$. The performance effects of $\mathrm{CHO}$ and fluid ingestion have been reported to be independent and additive (2), leading to the widespread application of sports drinks for training and competition. Some debate remains over the mechanisms behind this beneficial response, but the performance enhancement appears to be mediated through a combination of factors including the sparing of muscle and liver glycogen, preservation of circulating blood glucose levels and a maintenance of CHO utilisation late in exercise (12). Much of the work in this area has focused on long-duration exercise ( $>2$ hours), but there have also been reports of $\mathrm{CHO}$ ingestion enhancing the performance of relatively short-duration exercise where $\mathrm{CHO}$ availability is not traditionally thought to be limiting (24). This response has been attributed to a central effect of $\mathrm{CHO}$ ingestion, perhaps mediated through $\mathrm{CHO}$ sensing receptors found in the mouth acting as a feed-forward mechanism to prime the body for the delivery of substrate (6).

Paragraph 2 Many commercially-available CHO-electrolyte sports drinks contain carbohydrate at concentrations of around 6 to 8\%, and are approximately isotonic relative to human tissues. The $\mathrm{CHO}$ content of these beverages typically includes varying quantities of glucose, fructose, sucrose and maltodextrin. Jeukendrup and colleagues have shown that drinks containing mixtures of carbohydrate that contain both actively transported and passively absorbed carbohydrates may increase total 
exogenous carbohydrate oxidation during exercise and may in turn increase exercise capacity (23). There has been a recent trend for some sports drink manufacturers to release more dilute $\mathrm{CHO}$ beverages, typically marketed as a low energy option for the health and fitness industry. At present there are few studies investigating the efficacy of lower concentrations of $\mathrm{CHO}(0-6 \%)$ on exercise performance when all other factors have been standardised. Drinks with a low energy content result in faster gastric emptying times (40) and hypotonic carbohydrate-containing drinks may promote better water absorption from the small intestine than plain water or hypertonic CHO solutions (38). Taken together, it seems that a lower CHO concentration may be just as effective in enhancing exercise performance, particularly when fluid delivery is important for the maintenance of performance.

Paragraph 3 The capacity to perform prolonged exercise is reduced in a warm environment, with a progressive fall in time to fatigue as the ambient temperature increases $(17,35)$. Despite an understanding of the influence of ambient conditions on prolonged exercise capacity, the underlying mechanisms behind the deleterious effects of heat stress are still subject to much investigation $(7,29)$. Ingestion of fluids attenuates the increase in core temperature and heart rate, as well as the decline in cardiac output, normally observed during exercise in the heat (20,33). Consequently, a solution that can rapidly deliver fluid to the circulation may be more beneficial than a more concentrated $\mathrm{CHO}$ beverage during prolonged exercise in the heat. Supporting this suggestion, no benefit of $\mathrm{CHO}$ solutions over a CHO-free placebo were reported during exercise undertaken at $33^{\circ} \mathrm{C}$ (16). In addition, Galloway and Maughan (18) demonstrated that ingestion of a dilute glucose-electrolyte drink enhanced performance during exercise in the heat to a greater extent than ingestion of a smaller 
volume of a high-carbohydrate solution. Despite evidence suggesting that CHO availability is not a limiting factor to performance in warm conditions (35), several studies have provided convincing evidence of a benefit of either high $\mathrm{CHO}$ diets or CHO supplementation when undertaking prolonged exercise in the heat $(2,5,18,32,37)$.

Paragraph 4 The aim of this study was to examine the influence of dilute CHOelectrolyte solutions on physical performance undertaken in cool and warm conditions. To address this question, two parallel experiments were conducted, each involving the ingestion of a $0 \%, 2 \%, 4 \%$ and $6 \%$ CHO solution before and during prolonged exercise: one was undertaken at a work rate equivalent to $70 \%$ of $\mathrm{VO}_{2}$ peak at an ambient temperature of $10^{\circ} \mathrm{C}$, while subjects in the second study exercised at a work rate equivalent to $60 \%$ of $\mathrm{VO}_{2}$ peak at an ambient temperature of $30^{\circ} \mathrm{C}$.

\section{Methods}

Paragraph 5 This study consisted of two separate investigations involving fixedintensity exercise to volitional exhaustion as a measure of physical performance: one was undertaken in cool conditions $\left(10^{\circ} \mathrm{C}, 60 \%\right.$ relative humidity), and the second was completed in a warm environment $\left(30^{\circ} \mathrm{C}, 60 \%\right.$ relative humidity). Twelve healthy males volunteered to participate in each investigation (Cool: Age $22 \pm 2 \mathrm{y}$, Height 1.81 $\pm 0.08 \mathrm{~m}$, Body mass $73.5 \pm 8.1 \mathrm{~kg}, \mathrm{VO}_{2} \max 4.0 \pm 0.5 \mathrm{~L} / \mathrm{min}$; Warm: Age $21 \pm 2 \mathrm{y}$ Height $1.79 \pm 0.08 \mathrm{~m}$, Body mass $\left.80.2 \pm 7.1 \mathrm{~kg}, \mathrm{VO}_{2} \max 4.2 \pm 0.5 \mathrm{~L} / \mathrm{min}\right)$. All subjects were physically active and familiar with the sensation of strenuous exercise, but were 
not accustomed to exercise in a warm environment at the time of the study. Prior to volunteering all subjects received written details outlining the nature of the study. Following any questions regarding the protocol, a written statement of consent was signed. The protocol received prior approval from the Loughborough University Ethical Advisory Committee.

Paragraph 6 All subjects completed a preliminary test, a familiarisation trial and four experimental trials. Maximal oxygen uptake $\left(\mathrm{VO}_{2} \mathrm{max}\right)$ was first determined using a discontinuous, incremental exercise test to volitional exhaustion on a cycle ergometer (Monark E-series ergometer). The familiarisation trial followed the same format as the experimental trials. This was undertaken to ensure the subjects were accustomed to the procedures employed during the investigation and to minimise any potential learning or anxiety effects. During the familiarisation trials the appropriate volume of the carbohydrate-free test drink was ingested by the subject. To help ensure metabolic conditions were similar before each experimental trial, subjects were instructed to record dietary intake and physical activity during the day before the first trial and to replicate this in the day prior to the subsequent experimental trials. No alcohol consumption was permitted in the 24 hours before each trial, and subjects were also instructed to avoid strenuous exercise during this time.

Paragraph 7 Each subject completed four experimental trials, randomised and undertaken in a counter-balanced crossover manner. Trials were separated by at least five days. The solutions ingested consisted of a sugar-free fruit drink (Tesco Ltd, Cheshunt, UK), prepared to the manufacturer's guidelines: to this were added quantities of sucrose, glucose and fructose in a ratio of 50:25:25 to make final CHO 
concentrations of $2 \%(20 \mathrm{~g} / \mathrm{L}), 4 \%(40 \mathrm{~g} / \mathrm{L})$ and $6 \%(60 \mathrm{~g} / \mathrm{L})$. The solutions also contained 18mmol/L sodium and $2 \mathrm{mmol} / \mathrm{L}$ potassium. All drinks were maintained at a temperature of $21^{\circ} \mathrm{C}$ prior to ingestion. Trials took place after a fast of at least six hours. A radio-telemetry pill (HQ Inc, Palmetto, Florida, USA) was ingested 10 hours prior to exercise, to enable to measurement of core temperature. Subjects were instructed to ingest 500ml of plain water 90 minutes before commencing exercise. On arrival at the laboratory, subjects first emptied their bladder and a sample of urine was retained for measurement of osmolality (Gonotec Osmomat 030, YSI, Farnborough, UK). Post-void nude body mass was then measured, and a heart rate telemetry band positioned (Polar Vantage, Kempele, Finland).

Paragraph 8 Subjects entered the environmental chamber maintained at the appropriate conditions (cool $10^{\circ} \mathrm{C}, 60 \%$ relative humidity; warm $30^{\circ} \mathrm{C}, 60 \%$ relative humidity) and mounted a cycle ergometer before ingesting $3.0 \mathrm{~mL} / \mathrm{kg} \mathrm{BM}$ of the appropriate drink. Subjects then commenced exercise at a workload corresponding to $70 \% \mathrm{VO}_{2}$ peak in the cool study and $60 \% \mathrm{VO}_{2}$ peak in the warm study. The difference in workload between studies was intended to standardise the exercise duration between the two environmental conditions. Exercise continued until volitional exhaustion, defined as an inability to maintain a pedal cadence of 60 rev/min despite verbal encouragement from the experimenters.

Paragraph 9 Heart rate and core temperature were recorded every 10 minutes during exercise. Expired gas samples were collected and analysed at 15 minute intervals using the Douglas bag method. These data were used to estimate rates of substrate oxidation (36) and energy expenditure. Subjective ratings of perceived exertion (RPE; 
4) and thermal sensation (using a 21-point scale ranging from unbearable cold [-10] to unbearable heat [+10]) were assessed every 10 minutes. Subjects ingested $1.5 \mathrm{~mL} / \mathrm{kg}$ $\mathrm{BM}$ of the appropriate solution every 10 minutes during exercise. Following the completion of exercise subjects were reweighed to allow the estimation of sweat losses (30).

Paragraph 10 Statistical Analysis Direct statistical comparisons between the cool and warm studies were not undertaken due to the use of separate groups of volunteers and the difference in exercise intensity. Data are presented as mean \pm standard deviation (SD) unless otherwise stated. The differences in exercise time to exhaustion (TTE) and the magnitude of difference from the $0 \%$ trial were examined using a one-way repeated measures analysis of variance (ANOVA). A Bonferroni post hoc correction was applied to these data to maintain the family-wise error rate when examining differences between individual trials. Cohen's $d$ effect sizes (ES) for the differences in exercise time were also determined. To identify differences in data collected throughout each trial, two-way (time-by-trial) ANOVA were employed. Where a significant interaction was apparent, pair-wise differences were evaluated using the Bonferroni post hoc procedure. Based on the results of a previous investigation (28) we estimated a $90 \%$ probability of detecting a difference in TTE of 3.6 minutes with a sample size of 12 subjects (standardized ES $=0.12$; correlation between these repeat measures $\mathrm{r}=0.978, \mathrm{P}=0.001)$. 


\section{Results}

Study 1: $\operatorname{Cool}\left(10^{\circ} \mathrm{C}\right)$

Paragraph 11 There was main effect of trial apparent when examining the time to volitional exhaustion under each experimental condition $(P=0.012$; Figure 1A). There was a significant difference between trials when exercise capacity was expressed using the $0 \%$ trial as a baseline, with the mean difference from the time recorded during the $0 \%$ trial being $8.4 \pm 25.8 \mathrm{~min}(\mathrm{P}=0.427$; ES 0.20$)$ in the $2 \%$ trial, $19.4 \pm 19.5 \min (\mathrm{P}=0.032$; ES 0.72$)$ with the $4 \%$ solution and $21.5 \pm 25.2 \mathrm{~min}(\mathrm{P}=$ 0.044; ES 0.66) in the 6\% trial. While TTE was significantly longer in the $6 \%$ trial than the $2 \%$ trial (by $13.0 \pm 12.5$ min; $\mathrm{P}=0.025$ ), no other statistically significant differences were apparent between trials when $\mathrm{CHO}$ was ingested (all P > 0.05).

Paragraph 12 There were no differences apparent between trials in total sweat loss $(P=0.135)$ or the calculated sweat rate $(P=0.518)$ : sweat rates were $0.78 \pm 0.31 \mathrm{~L} / \mathrm{h}$, $0.85 \pm 0.36 \mathrm{~L} / \mathrm{h}, 0.77 \pm 0.19 \mathrm{~L} / \mathrm{h}$ and $0.76 \pm 0.26 \mathrm{~L} / \mathrm{h}$ in the $0 \%, 2 \%, 4 \%$ and $6 \%$ trials respectively. Resting heart rate was not different between trials, with a mean value of $79 \pm 6$ beats/min recorded prior to the start of exercise (Figure 2A). There was a clear elevation in heart rate during exercise $(\mathrm{P}<0.001)$, but the drink ingested did not alter this response $(\mathrm{P}=0.261)$. There was no difference in core temperature between trials prior to exercise (Figure 3A). While the $\mathrm{CHO}$ content of the drink ingested did not influence the core temperature response ( $\mathrm{P}=0.545$ ), there was a progressive increase during exercise in all trials ( $\mathrm{P}<0.001)$, reaching $38.1 \pm 0.5^{\circ} \mathrm{C}$ with the $0 \%$ drink, 38.1 $\pm 0.4^{\circ} \mathrm{C}$ in the $2 \%$ trial, $38.2 \pm 0.4^{\circ} \mathrm{C}$ with the $4 \%$ solution and $38.1 \pm 0.5^{\circ} \mathrm{C}$ in the $6 \%$ 
trial. Perceived exertion was not influenced by the drink ingested $(P=0.675)$, but there was a tendency for thermal stress to be higher on the $6 \%$ trial $(\mathrm{P}=0.062)$. A significant increase over time was apparent in all trials $(\mathrm{P}<0.001)$.

Paragraph 12 Expired gas data collected during the study are presented in Table 1. Oxygen uptake increased over time during exercise in each trial $(\mathrm{P}<0.001)$. The ingestion of the experimental solutions appeared to influence this response, with mean $\mathrm{VO}_{2}$ during exercise increasing with a higher concentration of $\mathrm{CHO}$ ingested, but this marginally failed to reach significance $(P=0.056)$. RER was not different between trials $(\mathrm{P}=0.294)$, but there was a significant reduction in RER as exercise progressed ( $\mathrm{P}<0.001)$. This response was greater during the $0 \%$ trial than during the trials where CHO was ingested. Rates of carbohydrate $(\mathrm{P}=0.244)$ and fat $(\mathrm{P}=0.136)$ oxidation during exercise were not influenced by the beverage ingested.

Study 2: Warm $\left(30^{\circ} \mathrm{C}\right)$

Paragraph 13. There was a significant difference between trials in TTE $(P=0.043$; Figure 1B). Further analysis of the performance data was undertaken by calculating the difference between the times recorded during each $\mathrm{CHO}$ trial and that attained during $0 \%$ trial. This was $9.6 \pm 16.0 \mathrm{~min}(\mathrm{P}=0.643$; ES 0.48) in the $2 \%$ trial, $10.9 \pm$ 12.1 min $(P=0.188$; ES 0.41) with the $4 \%$ solution and $17.6 \pm 21.3 \min (P=0.045$; ES 0.62) in the 6\% trial. There were no statistical differences in TTE apparent when comparisons were made between pairs of trials where $\mathrm{CHO}$ was ingested (all $\mathrm{P}>$ $0.05)$. 
Paragraph 14 There was a tendency for greater total sweat losses during exercise with higher drink $\mathrm{CHO}$ content $(\mathrm{P}=0.072)$, but there was no difference in the calculated sweat rate $(0 \% 1.51 \pm 0.32 \mathrm{~L} / \mathrm{h} ; 2 \% 1.46 \pm 0.31 \mathrm{~L} / \mathrm{h} ; 4 \% 1.46 \pm 0.27 \mathrm{~L} / \mathrm{h}$; $6 \% 1.46 \pm 0.32 \mathrm{~L} / \mathrm{h} ; \mathrm{P}=0.496)$. There was a clear elevation in heart rate during exercise $(\mathrm{P}<0.001)$, but the beverage ingested did not alter this response $(\mathrm{P}=0.464$; Figure 2B). There was no difference in core temperature between trials prior to exercise (Figure 3B). While the CHO content of the drink ingested did not influence the core temperature response $(\mathrm{P}=0.516)$, there was a progressive increase during exercise in all trials $(\mathrm{P}<0.001)$, reaching $38.4 \pm 0.5^{\circ} \mathrm{C} 38.8 \pm 0.7^{\circ} \mathrm{C}, 38.4 \pm 0.7^{\circ} \mathrm{C}$ and $38.5 \pm 0.7^{\circ} \mathrm{C}$ in the $0 \%, 2 \%, 4 \%$ and $6 \%$ trials respectively. While there was a significant increase in RPE and perceived thermal stress as exercise progressed observed during all trials $(\mathrm{P}<0.001)$, neither perceived exertion $(\mathrm{P}=0.802)$ or thermal stress $(\mathrm{P}=0.356)$ were influenced by the drink ingested.

Paragraph 15 Expired gas data collected during the warm study are presented in Table 2. Oxygen uptake increased during exercise in each trial $(\mathrm{P}<0.001)$, but was not influenced by the CHO content of the drink ingested $(\mathrm{P}=0.109)$. RER was not different between trials $(\mathrm{P}=0.226$ ), but there was a reduction in RER as exercise progressed in all trials $(\mathrm{P}=0.034)$. Rates of carbohydrate $(\mathrm{P}=0.105)$ and fat $(\mathrm{P}=$ 0.339) oxidation during exercise were not influenced by the drink ingested. 


\section{Discussion}

Paragraph 16 Most commercially available sports drinks have a CHO concentration of around 6-8\%. The formation of these beverages has been based on an abundance of published data supporting the benefits of $\mathrm{CHO}$ on exercise performance. However, evidence suggests that more dilute beverages empty rapidly from the stomach (40) and may promote better water absorption $(25,38)$. Consequently a lower carbohydrate concentration may be just as effective in enhancing exercise performance in some situations. This aim of the present study was to systematically investigate the effects of solutions containing between $0 \%$ and $6 \% \mathrm{CHO}$ on exercise performance in cool and warm environments.

Cool Study $\left(10^{\circ} \mathrm{C}\right)$

Paragraph 17 During prolonged exercise in temperate conditions lasting longer than 90 minutes, fatigue is characterised by low muscle glycogen availability and a fall in circulating blood glucose concentrations (12). The ingestion of exogenous CHO before and during exercise has been demonstrated to prolong exercise TTE $(8,9,13,19,28,31,39)$ through maintenance of circulating blood glucose levels and increased the rates of $\mathrm{CHO}$ utilisation late in exercise. The results of the present study demonstrate a significant improvement over the $0 \%$ trial when the $4 \%$ and $6 \% \mathrm{CHO}$ solutions were ingested. The difference in exercise capacity between the $0 \%$ and $6 \%$ trials was $21.5 \pm 24.2$ minutes, representing a 19.3\% improvement in TTE. As highlighted previously, a number of studies have reported similar findings when 
comparing a placebo to 6-8\% CHO solutions, but the performance effects of more dilute CHO solutions are not well defined. The 4\% CHO solution also resulted in an enhanced exercise capacity over that observed during the $0 \%$ trials $(+18.4$ minutes; $\mathrm{P}$ $=0.032$ ). The ingestion of $2 \% \mathrm{CHO}$ solution resulted in a mean increase in exercise capacity of 6.6 minutes over the $0 \%$ trial, the variability in this response meant this was not statistically significant $(\mathrm{P}=0.427$; ES 0.20). While there was a difference in TTE between the $2 \%$ and $6 \%$ trials $(\mathrm{P}=0.025$; ES 0.45$)$, no further statistical differences were apparent between the $\mathrm{CHO}$ trials, suggesting that when exercising in cool conditions a $4 \% \mathrm{CHO}$ solution may be just as effective at maintaining performance as a more concentrated $6 \%$ solution. Significant improvements in the performance of a pre-loaded time trial following the ingestion of relatively small quantities of glucose $(15,30$ and $60 \mathrm{~g} / \mathrm{h})$ compared to a $\mathrm{CHO}$-free placebo has also been recently reported (39).

Paragraph 18 No differences were observed in the estimated rates of $\mathrm{CHO}$ and fat oxidation. CHO ingestion is widely reported to enhance overall $\mathrm{CHO}$ oxidation rate, while also suppressing lipolysis, and consequently attenuating the rate of fat oxidation $(12,26)$. Several other investigators have reported the absence of differences in rates of $\mathrm{CHO}$ and fat oxidation with ingestion of 6 and 8\% CHO solutions $(31,34)$. The difference between studies may be explained by the quantities of CHO administered, with the absence of response potentially related to the relatively small amount of CHO ingested in the present study. Studies reporting a marked effect of $\mathrm{CHO}$ ingestion on rates of $\mathrm{CHO}$ and fat oxidation have typically administered $60-75 \mathrm{~g}$ $\mathrm{CHO} /$ hour (10), whereas subjects in the present study ingested around $14 \mathrm{~g}$, $29 \mathrm{~g}$ or 43g $\mathrm{CHO} /$ hour during the $2 \%, 4 \%$ and $6 \%$ trials respectively (assuming the ingestion 
of $\sim 120 \mathrm{ml}$ every 10 minutes for an $80 \mathrm{~kg}$ subject). This view is supported by recent data demonstrating no change in total $\mathrm{CHO}$ oxidation following the ingestion of glucose at a rate of $30 \mathrm{~g} / \mathrm{h}$, but a significant increase compared to a placebo control was apparent when $60 \mathrm{~g} / \mathrm{h} \mathrm{CHO}$ was ingested (39). These data also suggest that higher rates of $\mathrm{CHO}$ ingestion result in a reduction in the utilisation of endogenous glucose from the liver, with no change in muscle glycogen utilisation. Maximal rates of exogenous glucose oxidation during exercise are typically reported at 1.0-1.1 g/min, while sugars requiring hepatic metabolism prior to muscle utilisation (e.g. fructose and galactose) appear to be limited to around $0.4-0.7 \mathrm{~g} / \mathrm{min}$ (26). The delivery of ingested $\mathrm{CHO}$ to the muscle appears to be a limiting factor in determining exogenous CHO oxidation rates. Recent evidence suggests that the inclusion of multiple transportable substrates, as employed in the present study, can increase the rate of CHO delivery to the circulation and may enhance oxidation rates beyond 1.0$1.1 \mathrm{~g} / \mathrm{min}(22)$.

Paragraph 19 There was no significant effect of the treatment on the subjects' thermoregulatory response to the exercise: no differences between trials were apparent in core temperature, although there was a tendency for perceived thermal stress to be higher throughout the $6 \%$ trial than on the other trials. Similar responses have been reported by Galloway and Maughan (2000) following the ingestion of CHO solutions during exercise. 
Warm study $\left(30^{\circ} \mathrm{C}\right)$

Paragraph 20 The capacity to perform prolonged exercise is reduced in a warm environment $(17,35)$, but the underlying mechanisms behind this response are still poorly understood. Potential peripheral mechanisms include impaired substrate availability or utilization, accumulation of metabolic waste products, or the loss of body fluids, but these do not adequately explain this reduction in performance, leading to the suggestion that the CNS may be important (29). Despite evidence suggesting that substrate availability is not limiting, several studies have reported improvements in performance following the ingestion of a high CHO diet (37) and the use of CHO supplements before and during exercise $(2,5,18,32)$. Certainly the ingested fluid attenuates the increase in core temperature and heart rate typically observed during exercise in the heat (20,33). Consequently, a solution that can rapidly deliver fluid to the circulation is likely to be more beneficial than a concentrated CHO-containing fluid during prolonged exercise in the heat (18).

Paragraph 21 This line of thought is supported by data presented by Febbraio and colleagues (16) demonstrating no difference in exercise TTE compared to a control condition when $4.2 \%, 7 \%$ and $14 \% \mathrm{CHO}$ solutions were ingested during exercise undertaken at $33^{\circ} \mathrm{C}$. There was a tendency for exercise capacity to be reduced at the highest drink CHO concentration (14\%), and this was attributed by the authors to a greater reduction in plasma volume and a faster rate of core temperature rise resulting from a slower rate of gastric emptying and intestinal absorption. Perhaps contrary to these findings, the present study reported longer exercise times with increasing $\mathrm{CHO}$ concentration (Figure 1B), although this effect reached statistical significance only 
when comparing the $0 \%$ and $6 \%$ trials. In fact, ingestion of the $6 \% \mathrm{CHO}$ solution resulted in a $18.6 \%$ improvement in exercise capacity over the $0 \%$ trial, which is strikingly similar to the difference between these two trials observed in the cool study. It is worth noting that the highest beverage $\mathrm{CHO}$ concentration (6\%) employed in the present study is lower than the $14-15 \%$ used in previous reports $(16,18)$.

Paragraph 22 Several studies have reported an increase in total CHO oxidation when exercise is performed in warm conditions when compared to exercise in a temperate environment $(15,16,22)$. This response appears to be caused by increased rates of muscle glycogen oxidation (+25\%), and is accompanied by a reduction in exogenous CHO usage (22). Although the rate muscle glycogen utilisation is increased during exercise undertaken in warm conditions (15), relatively high muscle glycogen concentrations have been reported at the point of fatigue (35). Despite the apparent reduction in exogenous $\mathrm{CHO}$ oxidation rates, the present study and several previous publications have reported a benefit of ingesting $\mathrm{CHO}$ solutions before and during prolonged exercise in a warm environment $(2,5,32)$. In the absence of clear metabolic differences between trials, it is possible that these effects of $\mathrm{CHO}$ may reflect a central effect of $\mathrm{CHO}$ ingestion, mediated through $\mathrm{CHO}$ sensing receptors found in the mouth (6) or elsewhere.

Paragraph 23 In a similar manner to the findings of the cool study, the ingestion of CHO did not produce a measureable change to the physiological response to exercise in the heat; there were no detectable differences in heart rate, core temperature or rates of substrate utilisation. There was a tendency for the sweat losses incurred during exercise to be higher with the increasing $\mathrm{CHO}$ content of the drink, but this is 
was caused by the increased exercise duration: the calculated sweat rate was not different between trials $(\mathrm{P}=0.688)$. Fatigue during prolonged exercise in the heat has been attributed to the attainment of a critical core temperature, resulting in a loss of drive and motivation to continue exercise. This has been suggested to act as a safety mechanism, limiting heat production and preserving the integrity of the organism in the presence of heat stress. It seems that this view is perhaps overly simplistic, with feedback to the CNS arising from several sources including skin temperature receptors, heart rate, and central venous pressure resulting in a deterioration of CNS drive with exercise-induced hyperthermia. A key role for skin temperature in influencing performance in the heat has been proposed (7). Weighted mean skin temperature was not monitored in the present study, but it appears unlikely that any differences between trials would have been apparent.

Paragraph 24 The validity, reliability and sensitivity of tests commonly employed as a measure of exercise performance has generated considerable controversy in recent years. Constant power tests to volitional exhaustion have been employed to examine the influence of various interventions on performance, but this method of testing has been criticised for a lack of ecological validity and poor test-retest reliability. This view is supported by the findings of Jeukendrup and colleagues (27), who found a large day-to-day variability (CV 27\%) in TTE tests, and a much smaller variability in a time trial protocol $(<4 \%)$. While some continue to voice concerns over a lack of ecological validity, data from our research group report more consistent performance in time to fatigue tests (28; CV 6\%) and recent reports have highlighted similar errors of measurement when changes in performance are normalised across tests (21). A key factor to consider when selecting an appropriate exercise test is its sensitivity, and the 
smallest worthwhile effect that can be reliability detected (14). Amann and colleagues (1) demonstrated that TTE and time trial protocols display a similar sensitivity to the effects of hypoxia and hyperoxia on performance, and suggest that this finding will extend to other factors influencing performance. This is brought about by larger effects on performance in response to an intervention with constant power tests than are typically observed in time trial protocols: this compensates for the larger testretest variability, resulting in a very similar signal-to-noise ratio to that seen with time trial protocols $(1,14)$. In some research situations, the obvious limitation of time trialtype test is a difficulty in comparing the effect of an intervention on the physiological response to exercise, since at any given time one volunteer's relative workload may vary greatly from that of other participants. Although this can be overcome through the addition of a period of constant load exercise undertaken before the time trial, as described by Jeukendrup and colleagues (27).

Paragraph 25 In conclusion, compared to a CHO-free placebo, ingestion of solutions containing 4\% and 6\% CHO improved exercise performance in a cool environment, whereas a $6 \% \mathrm{CHO}$ beverage resulted in a significant increase in TTE at $30^{\circ} \mathrm{C}$. Discussion of the responses observed in the cool and warm studies has been kept largely separate due to the use of two groups of volunteers and different exercise intensities, but some clear comparisons can be drawn. In particular, the magnitude of change observed between the $0 \%$ and $6 \%$ trials in each environmental condition was remarkably similar ( 19\%), given the apparently different mechanisms of fatigue thought to be in operation. Despite the differences in exercise performance, there were few alterations to the physiological responses to the exercise in both environmental 
conditions. This is perhaps due to the relatively small quantity of CHO ingested, compared to some previous investigations.

\section{Acknowledgements}

The authors would like to thank David Ferguson, Astin Ewington, Floor van Langen and John Quigley for their technical assistance during this investigation. The study was carried out in relation to the product Powerade and was funded in part by The Coca-Cola Company. The results of the present study do not constitute endorsement by ACSM.

\section{References}

1. Amann M, Hopkins WG, Marcora SM. Similar sensitivity of time to exhaustion and time-trial time to changes in endurance. Med Sci Sports Exerc 2008;40:5748.

2. Below PR, Mora-Rodriguez R, Gonzalez-Alonso J, Coyle EF. Fluid and carbohydrate ingestion independently improve performance during $1 \mathrm{~h}$ of intense exercise. Med Sci Sports Exerc. 1995;27:200-210.

3. Björkman O, Sahlin K, Hagenfeldt L, Wahren J. Influence of glucose and fructose ingestion on the capacity for long-term exercise in well-trained men. Clin Physiol. 1984;4:483-494. 
4. Borg GA. Psychophysical bases of perceived exertion. Med Sci Sports Exerc 1982;14:377-81.

5. Carter JM, Jeukendrup AE, Mundel T, Jones DA. Carbohydrate supplementation improves moderate and high-intensity exercise in the heat. Pflugers Arch. 2003;446:211-9.

6. Carter JM, Jeukendrup AE, Jones DA. The effect of carbohydrate mouth rinse on 1-h cycle time trial performance. Med Sci Sports Exerc. 2004;36:2107-11.

7. Cheuvront SN, Kenefick RW, Montain SJ, Sawka MN. Mechanisms of aerobic performance impairment with heat stress and dehydration. J Appl Physiol. 2010;109:1989-95.

8. Coggan AR, Coyle EF. Reversal of fatigue during prolonged exercise by carbohydrate infusion or ingestion. J Appl Physiol 1987;63:2388-2395.

9. Coggan AR, Coyle EF. Metabolism and performance following carbohydrate ingestion late in exercise. Med Sci Sports Exerc. 1989;21:59-65.

10. Coggan AR, Swanson SC. Nutritional manipulations before and during endurance exercise: effects on performance. Med Sci Sports Exerc. 1992;24:S331-335.

11. Convertino VA, Armstrong LE, Coyle EF, Mack GW, Sawka MN, Senay LC, Sherman WM. ACSM position stand: exercise and fluid replacement. Med Sci Sports Exerc. 1996;28: i-ix.

12. Coyle EF. Fluid and fuel intake during exercise. J Sports Sci. 2004;22:39-55.

13. Coyle EF, Coggan AR, Hemmert MK, Ivy JL. Muscle glycogen utilization during prolonged strenuous exercise when fed carbohydrate. J Appl Physiol. 1986;61:165-172. 
14. Currell K, Jeukendrup AE. Validity, reliability and sensitivity of measures of sporting performance. Sports Med. 2008;38:297-316.

15. Febbraio MA, Snow RJ, Stathis CG, Hargreaves M, Carey MF. Effect of heat stress on muscle energy metabolism during exercise. J Appl Physiol. 1994;77:2827-2831.

16. Febbraio MA, Murton P, Selig SE, Clark SA, Lambert DL, Angus DJ, Carey MF. Effect of CHO ingestion on exercise metabolism and performance in different ambient temperatures. Med Sci Sports Exerc. 1996;28:1380-1387.

17. Galloway SDR, Maughan RJ. Effects of ambient temperature on the capacity to perform prolonged cycle exercise in man. Med Sci Sports Exerc. 1997;29:12401249.

18. Galloway SDR, Maughan RJ. The effects of substrate and fluid provision on thermoregulatory and metabolic responses to prolonged exercise in a hot environment. J Sports Sci. 2000;18:339-351.

19. Gleeson M, Maughan RJ \& Greenhaff PL. Comparison of the effects of preexercise feeding of glucose, glycerol and placebo on endurance and fuel homeostasis in man. Eur J Appl Physiol. 1986;55:645-653.

20. Hamilton MT, Gonzalez-Alonso J, Montain SJ, Coyle EF. Fluid replacement and glucose infusion during exercise prevent cardiovascular drift. J Appl Physiol. $1991 ; 71: 871-7$

21. Hinckson EA, Hopkins WG. Reliability of time to exhaustion analyzed with critical-power and log-log modelling. Med Sci Sports Exerc. 2005;37:696-701.

22. Jentjens RLPG, Achten J, Jeukendrup AE. High oxidation rates from combined carbohydrates ingested during exercise. Med Sci Sports Exerc. 2004;36:15511558. 
23. Jeukendrup AE. Carbohydrate and exercise performance: the role of multiple transportable carbohydrates. Curr Opinion Clin Nutr Metab Care. 2010;13:452457.

24. Jeukendrup A, Brouns F, Wagenmakers AJ, Saris WH. (1997) Carbohydrateelectrolyte feedings improve $1 \mathrm{~h}$ time trial cycling performance. Int J Sports Med. $18,125-9$.

25. Jeukendrup AE, Currell K, Clarke J, Cole J, Blannin AK. Effect of beverage glucose and sodium content on fluid delivery. Nutr Metab (Lond). 2009;20:6-9.

26. Jeukendrup AE, Jentjens R. Oxidation of carbohydrate feedings during prolonged exercise: current thoughts, guidelines and directions for future research. Sports Med. 2000;29:407-24.

27. Jeukendrup A, Saris WH, Brouns F, Kester AD. A new validated endurance performance test. Med Sci Sports Exerc. 1996;28:266-70

28. Maughan RJ, Fenn CE, Leiper JB. Effects of fluid, electrolyte and substrate ingestion on endurance capacity. Eur J Appl Physiol Occup Physiol. 1989;58:481-6.

29. Maughan RJ, Shirreffs SM, Watson P. Exercise, heat, hydration and the brain. $J$ Am Coll Clin Nutr. 2007;26:604S-612S.

30. Maughan RJ, Shirreffs SM, Leiper JB. Errors in the estimation of sweat loss and changes in hydration status from changes in body mass during exercise. $J$ Sports Sci. 2007;25:797-804.

31. McConnell G, Snow RJ, Proietto J, Hargreaves M Muscle metabolism during prolonged exercise in humans: influence of carbohydrate availability. J Appl Physiol. 1999;87:1083-1086. 
32. Millard-Stafford ML, Sparling PB, Rosskopf LB, Snow TK. Should the carbohydrate concentration of a sports drink be less than 8\% during exercise in the heat? Int J Sport Nutr Exerc Metab. 2005;15:117-30.

33. Montain SJ, Coyle EF. Influence of graded dehydration on hyperthermia and cardiovascular drift during exercise. J Appl Physiol. 1992;73:1340-50.

34. Murray R, Seifert JG, Eddy DE, Paul GL, Halaby GA. Carbohydrate feeding and exercise: effect of beverage carbohydrate content. Eur J Appl Physiol. 1989;59:152-8.

35. Parkin, J.M., Carey, M.F., Zhao, S. \& Febbraio, M.A. Effect of ambient temperature on human skeletal muscle metabolism during fatiguing submaximal exercise. J Appl Physiol. 1999;86:902-908.

36. Peronnet F, Massicotte D. Table of nonprotein respiratory quotient: an update. Can J Sports Sci. 1991;16:23-29.

37. Pitsiladis YP, Maughan RJ. The effects of exercise and diet manipulation on the capacity to perform prolonged exercise in the heat and in the cold in trained humans. J Physiol. 1999;517:919-30.

38. Rehrer NJ, Wagenmakers AJ, Beckers EJ, Halliday D, Leiper JB, Brouns F, Maughan RJ, Westerterp K, Saris WH. Gastric emptying, absorption, and carbohydrate oxidation during prolonged exercise. J Appl Physiol. 1992;72:46875.

39. Smith JW, Zachwieja JJ, Péronnet F, Passe DH, Massicotte D, Lavoie C, Pascoe DD. Fuel selection and cycling endurance performance with ingestion of [13C]glucose: evidence for a carbohydrate dose response. J Appl Physiol. 2010;108:1520-9. 
40. Vist GE, Maughan RJ. The effect of osmolality and carbohydrate content on the rate of gastric emptying of liquids in man. $J$ Physiol. 1995;486:523-31. 


\section{Figure legends}

Figure 1: Time to exhaustion in the four experimental trials undertaken in $10^{\circ} \mathrm{C}(\mathrm{A})$ and $30^{\circ} \mathrm{C}(\mathrm{B}) .{ }^{*}$ denotes a significant difference in time to exhaustion from the $0 \%$ trial $(\mathrm{P}<0.05)$.

Figure 2: Heart rate at rest and during exercise in $10^{\circ} \mathrm{C}(\mathrm{A})$ and $30^{\circ} \mathrm{C}(\mathrm{B})$. To improve clarity, data is presented as mean \pm group SD. Where significant differences are present, these are described in the text.

Figure 3: Core temperature at rest and during exercise in $10^{\circ} \mathrm{C}(\mathrm{A})$ and $30^{\circ} \mathrm{C}(\mathrm{B})$. To improve clarity, data is presented as mean \pm group SD. Where significant differences are present, these are described in the text. 

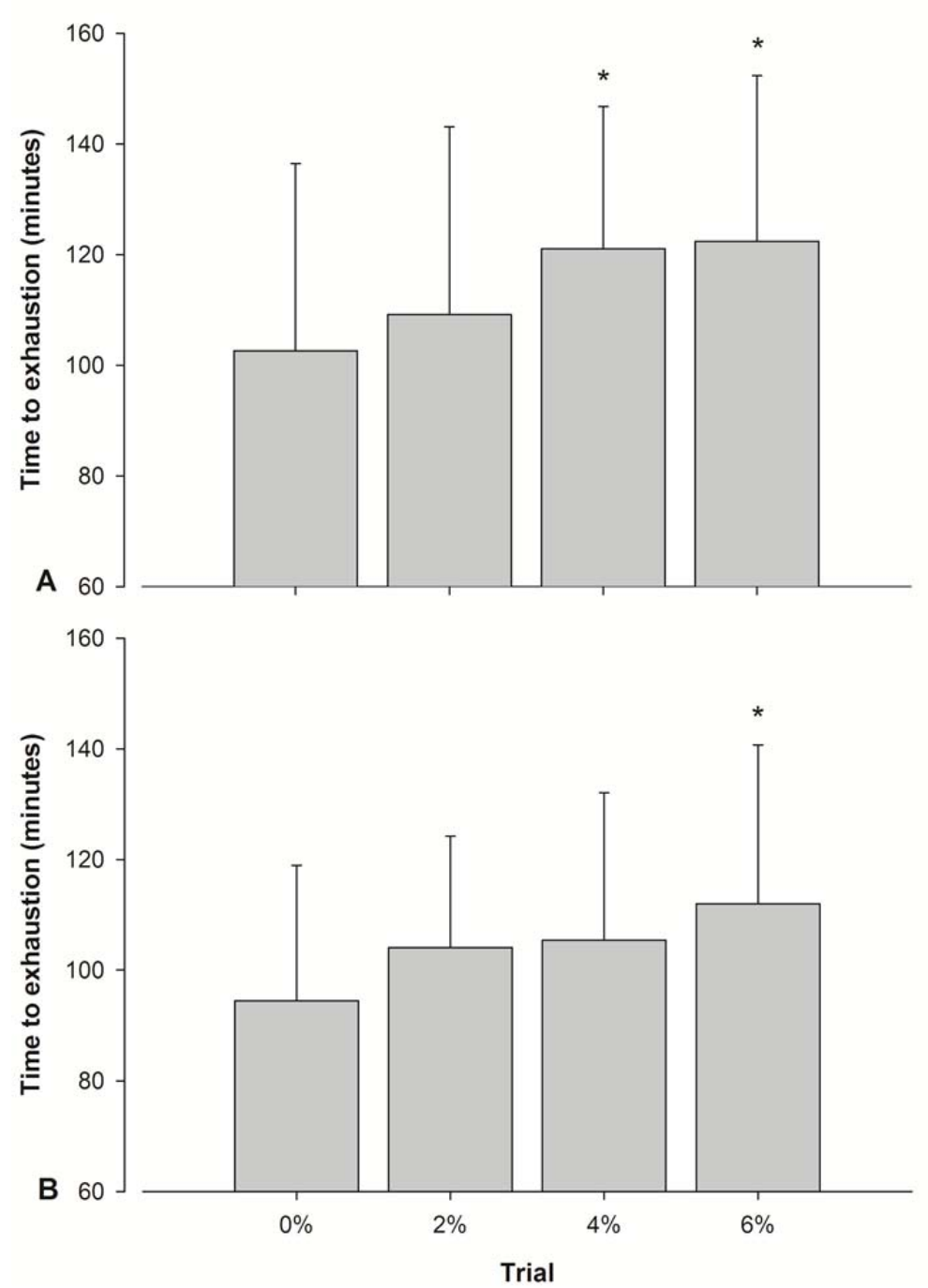


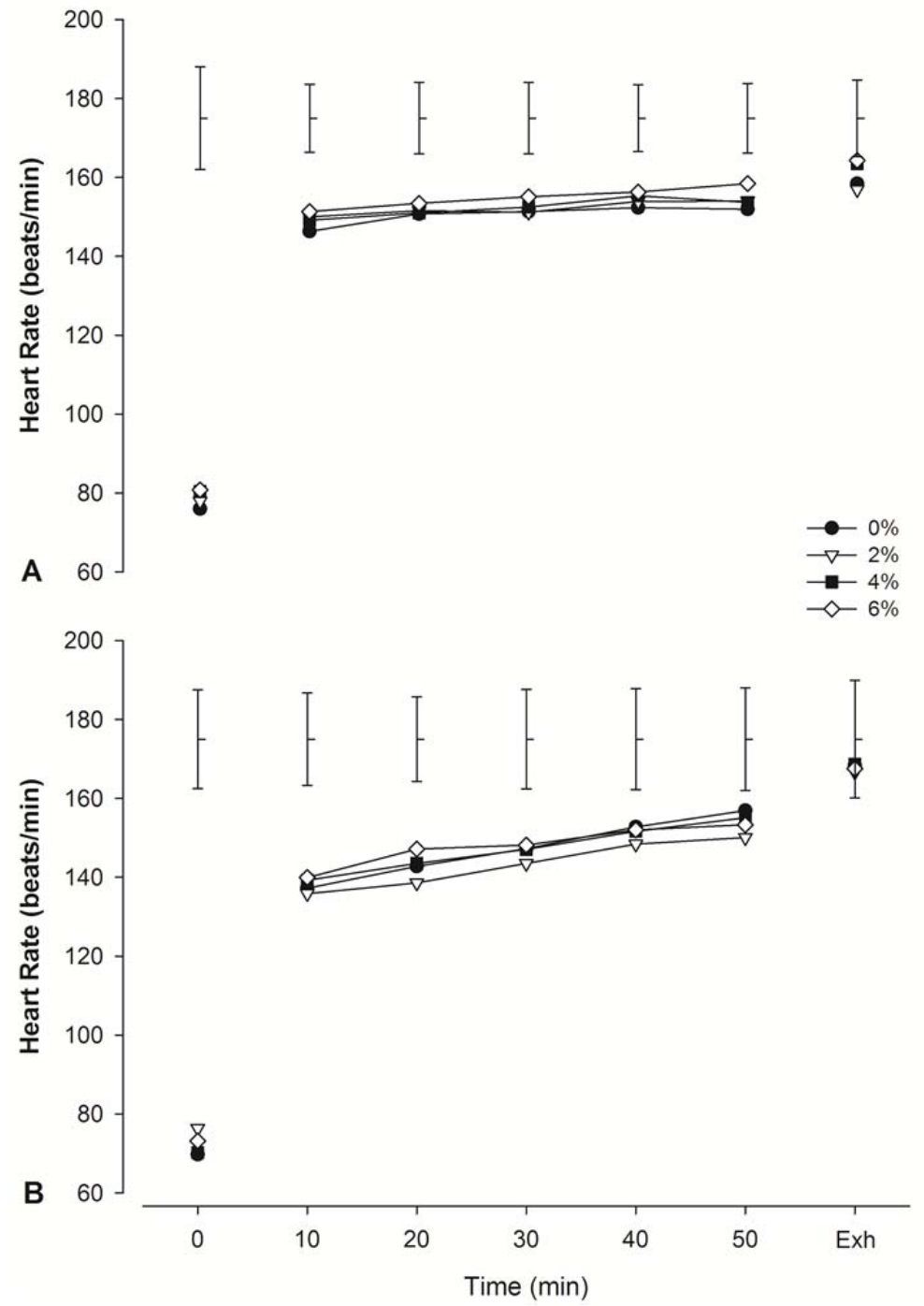




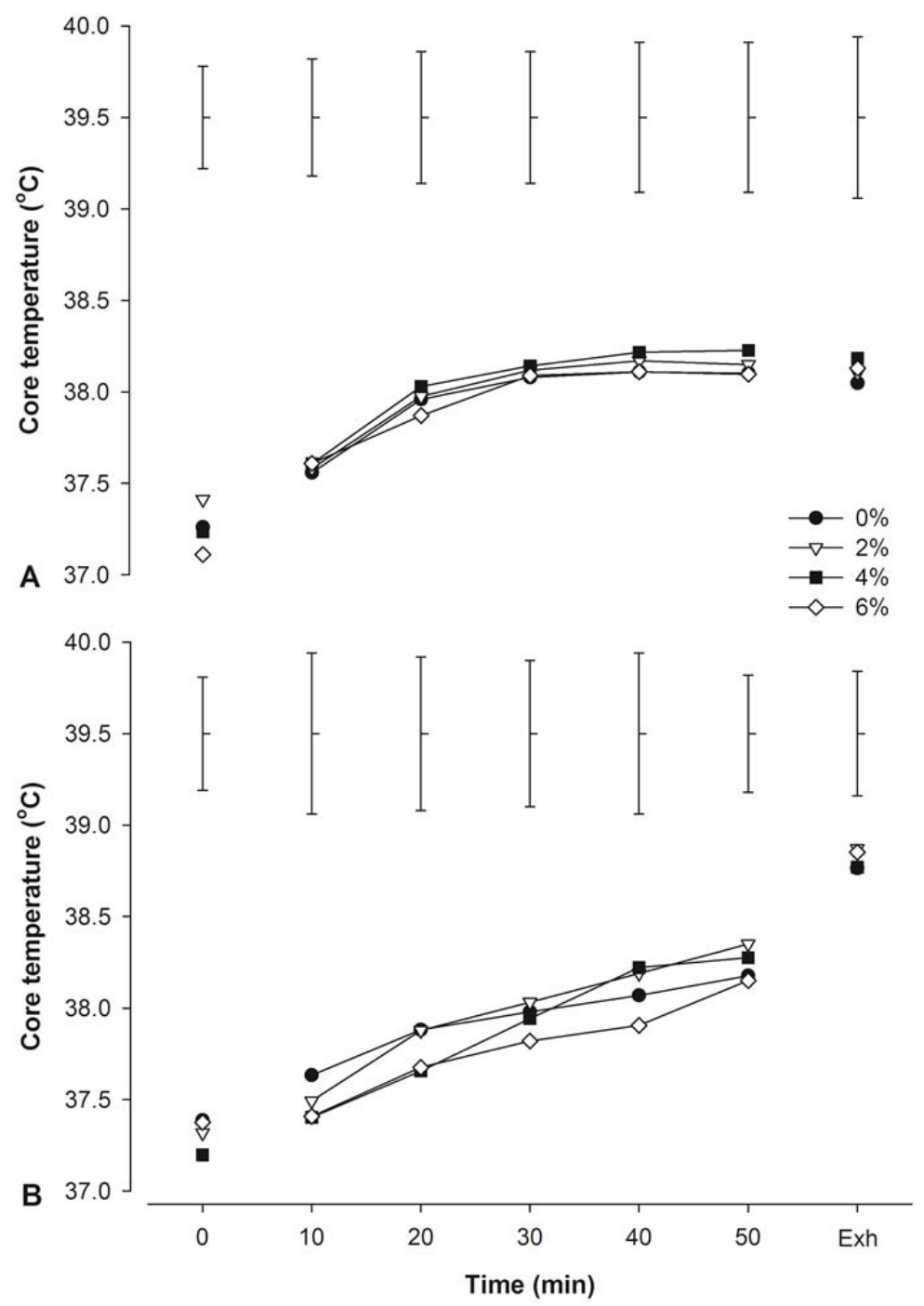


Table 1: Oxygen uptake (VO2), respiratory exchange ratio (RER) and estimated rates of carbohydrate and fat oxidation after 15, 30 and 45 minutes of exercise and at the point of fatigue at $10^{\circ} \mathrm{C}$.

\begin{tabular}{|c|c|c|c|c|}
\hline & 15 & 30 & 45 & $\mathbf{E x}$ \\
\hline \multicolumn{5}{|c|}{ Oxygen Uptake (L/min) } \\
\hline $0 \%$ & $3.08 \pm 0.47$ & $3.08 \pm 0.53$ & $3.15 \pm 0.47$ & $3.45 \pm 0.43$ \\
\hline $2 \%$ & $3.02 \pm 0.46$ & $3.20 \pm 0.37$ & $3.31 \pm 0.45$ & $3.31 \pm 0.42$ \\
\hline $4 \%$ & $3.08 \pm 0.43$ & $3.08 \pm 0.40$ & $3.19 \pm 0.40$ & $3.55 \pm 0.34$ \\
\hline $6 \%$ & $3.13 \pm 0.45$ & $3.22 \pm 0.52$ & $3.35 \pm 0.47$ & $3.58 \pm 0.42$ \\
\hline \multicolumn{5}{|c|}{ Respiratory Exchange Ratio (no units) } \\
\hline $0 \%$ & $0.97 \pm 0.04$ & $0.95 \pm 0.04$ & $0.94 \pm 0.04$ & $0.89 \pm 0.03$ \\
\hline $2 \%$ & $0.97 \pm 0.03$ & $0.95 \pm 0.04$ & $0.95 \pm 0.03$ & $0.92 \pm 0.03$ \\
\hline $4 \%$ & $0.98 \pm 0.05$ & $0.97 \pm 0.05$ & $0.95 \pm 0.03$ & $0.92 \pm 0.05$ \\
\hline $6 \%$ & $0.95 \pm 0.03$ & $0.94 \pm 0.03$ & $0.94 \pm 0.03$ & $0.93 \pm 0.03$ \\
\hline
\end{tabular}

CHO oxidation rate ( $\mathrm{g} / \mathrm{min})$

$\begin{array}{lllll}0 \% & 3.7 \pm 0.7 & 3.4 \pm 0.9 & 3.3 \pm 0.9 & 3.1 \pm 0.7 \\ 2 \% & 3.8 \pm 0.6 & 3.7 \pm 0.7 & 3.7 \pm 0.6 & 3.1 \pm 0.6 \\ 4 \% & 3.9 \pm 1.0 & 3.8 \pm 1.1 & 3.6 \pm 0.7 & 3.7 \pm 0.7 \\ 6 \% & 3.6 \pm 0.5 & 3.4 \pm 0.6 & 3.6 \pm 0.5 & 3.6 \pm 0.5\end{array}$

Fat oxidation rate $(\mathrm{g} / \mathrm{min})$

$\begin{array}{lllll}0 \% & 0.2 \pm 0.2 & 0.3 \pm 0.2 & 0.3 \pm 0.2 & 0.7 \pm 0.2 \\ 2 \% & 0.1 \pm 0.2 & 0.2 \pm 0.3 & 0.3 \pm 0.2 & 0.5 \pm 0.3 \\ 4 \% & 0.1 \pm 0.3 & 0.1 \pm 0.3 & 0.2 \pm 0.2 & 0.4 \pm 0.3 \\ 6 \% & 0.3 \pm 0.2 & 0.3 \pm 0.2 & 0.3 \pm 0.2 & 0.4 \pm 0.2\end{array}$


Table 2: Oxygen uptake (VO2), respiratory exchange ratio (RER) and estimated rates of carbohydrate and fat oxidation after 15, 30 and 45 minutes of exercise and at the point of fatigue at $30^{\circ} \mathrm{C}$.

\section{Oxygen Uptake (L/min)}

$\begin{array}{lllll}0 \% & 2.64 \pm 0.56 & 2.82 \pm 0.35 & 2.85 \pm 0.31 & 3.10 \pm 0.30 \\ 2 \% & 2.78 \pm 0.35 & 2.98 \pm 0.57 & 3.01 \pm 0.57 & 3.19 \pm 0.42 \\ 4 \% & 2.80 \pm 0.44 & 2.94 \pm 0.45 & 2.87 \pm 0.35 & 3.30 \pm 0.46 \\ 6 \% & 2.77 \pm 0.43 & 2.88 \pm 0.41 & 2.94 \pm 0.47 & 3.16 \pm 0.45\end{array}$

\section{Respiratory Exchange Ratio (no units)}

$\begin{array}{lllll}0 \% & 0.93 \pm 0.05 & 0.93 \pm 0.05 & 0.92 \pm 0.06 & 0.90 \pm 0.06 \\ 2 \% & 0.96 \pm 0.05 & 0.95 \pm 0.04 & 0.94 \pm 0.03 & 0.92 \pm 0.04 \\ 4 \% & 0.97 \pm 0.05 & 0.95 \pm 0.06 & 0.95 \pm 0.05 & 0.93 \pm 0.05 \\ 6 \% & 0.97 \pm 0.05 & 0.94 \pm 0.03 & 0.95 \pm 0.05 & 0.96 \pm 0.07\end{array}$

CHO oxidation rate ( $\mathrm{g} / \mathrm{min})$

$\begin{array}{lllll}0 \% & 3.0 \pm 0.9 & 3.0 \pm 0.6 & 2.9 \pm 0.5 & 2.8 \pm 1.0 \\ 2 \% & 3.1 \pm 0.5 & 3.2 \pm 0.7 & 3.2 \pm 0.7 & 3.1 \pm 0.5 \\ 4 \% & 3.3 \pm 0.5 & 3.2 \pm 0.5 & 3.3 \pm 0.5 & 3.3 \pm 0.8 \\ 6 \% & 3.1 \pm 0.6 & 3.1 \pm 0.5 & 3.2 \pm 0.7 & 3.3 \pm 0.7\end{array}$

Fat oxidation rate $(\mathrm{g} / \mathrm{min})$

$\begin{array}{lllll}0 \% & 0.3 \pm 0.2 & 0.3 \pm 0.2 & 0.4 \pm 0.3 & 0.6 \pm 0.3 \\ 2 \% & 0.2 \pm 0.2 & 0.3 \pm 0.2 & 0.3 \pm 0.2 & 0.4 \pm 0.3 \\ 4 \% & 0.2 \pm 0.2 & 0.3 \pm 0.2 & 0.3 \pm 0.2 & 0.4 \pm 0.3 \\ 6 \% & 0.2 \pm 0.2 & 0.3 \pm 0.2 & 0.3 \pm 0.3 & 0.3 \pm 0.2\end{array}$


